Original Research Article

\title{
Correlation of B-scan, CT scan and biopsy findings in orbital masses (space occupying lesions)
}

\author{
Pandarpurkar M. ${ }^{1}$, Shilpa G.V. ${ }^{2}$, Amruthavalli K.S. ${ }^{3}$, N. Rajesh Kumar ${ }^{4}$ \\ ${ }^{1}$ Dr. Modini Pandarpurkar, Professor and HOD, ${ }^{2}$ Dr G.V. Shilpa, Assistant Professor, ${ }^{3}$ Dr. K.S. Amruthavalli, Senior Resident, \\ ${ }^{4}$ Dr. N. Rajesh Kumar, Junior Resident, all authors are affiliated with Department of Ophthalmology, Sarojinidevi Eye Hospital, \\ Osmania Medical College, Hyderabad, Telangana, India.
}

Corresponding Author: Dr. K.S. Amruthavalli, Senior Resident, Department of Ophthalmology, Sarojinidevi Eye Hospital, Osmania Medical College, Hyderabad, Telangana, India. Email: dramruthaoph@gmail.com

\begin{abstract}
Introduction: Orbital masses or space occupying lesions, involving the orbit, produce symptoms and signs by compression, infiltration and/or infarction of orbital structures. A wide variety of processes can produce space-occupying lesions in and around the orbit. Imaging can be done to precisely localize a lesion, to help establish a diagnosis or generate a differential diagnosis that guides management. Material and Methods: Over a period of 18 months, patients with space occupying lesion of the orbit, in the age group of 1 to 70 years are included in the study. Proptosis assessment was done. Results: All the patients were subjected to B scan, CT scan and biopsy. On comparing the findings of B-Scan, CT Scan and biopsy (biopsy findings being taken as gold standard), B-Scan accurately diagnosed $83.33 \%$ of the cases, where as CT scan diagnosed only $60 \%$ of the cases accurately. Rest of the cases, there was no correlation between the B-Scan/CT scan and biopsy. Conclusions: B-Scan appears to be the better diagnosing tool in identifying most of the orbital lesions when compared to the CT scan. Considering radiation exposure, repeated examination, cost effectiveness and time consumption, B-Scan is advantageous over CT scan in the initial work up and follow up of cases.
\end{abstract}

Keywords: Orbital masses, Orbital mass biopsy, B-Scan, CT Scan, Benign, Malignant orbital lesions

\section{Introduction}

Orbital masses or space occupying lesions, involving the orbit, produce symptoms and signs by compression, infiltration and/or infarction of orbital structures [1]. A wide variety of processes can produce space-occupying lesions in and around the orbit. These include benign neoplasms malignant neoplasms, vascular lesions, inflammatory disease, congenital lesions and infection among other causes to follow a known lesion for progression, or some combination of these [2].

Imaging can be done to precisely localize a lesion, to help establish a diagnosis or generate a differential diagnosis that guides management.

Ultrasound has become an extremely useful tool in assessment of orbital lesions since it is efficient, inexpensive, non-invasive and can be repeatedly performed in order to evaluate treatment effectiveness or disease progression. With understanding of the indications for the B-scan, and the proper technique, a vast amount of

Manuscript received: $20^{\text {th }}$ October 2019

Reviewed: $30^{\text {th }}$ October 2019

Author Corrected: $6^{\text {th }}$ November 2019

Accepted for Publication: $12^{\text {th }}$ November 2019

Tropical Journal of Ophthalmology and Otolaryngology information can be gathered, which cannot be obtained by the clinical examination alone. The superficial location of the eye and its cystic composition make ultrasound ideal for the imaging of the eye. It can be safely performed as an outpatient procedure without use of any anaesthetic or sedatives. It is highly useful, especially in the presence of opaque media. It is non-hazardous, atraumatic and invaluable in the evaluation of orbito-ocular diseases [3].

For orbital imaging in the acute setting, computerized tomography (CT Scan) is the first line modality. CT scan is valuable, efficient imaging test that uses thin serial $\mathrm{X}$ ray images processed by computer. CT scan allows us to discern the location, extent and the configuration of the lesion, and its effect on the adjacent structures. It also allows us for understanding the possible tissue mass composition. Contrast study involves imaging of the particular area, after injection of the radiological contrast medium intravenously. CT Scan is helpful in assessment of proptosis, size of the tumor, circumscription of the tumor, shape and margin of the tumor, effect on the surrounding structures, internal consistency, surrounding bone, exact location and extra orbital extension of the tumor [4]. 
With each imaging pattern having its own differential diagnosis, orbital mass lesions often pose a diagnostic challenge. To provide accurate, specific, and sufficiently comprehensive diagnosis, to optimize the clinical management, and to estimate prognosis, pathological examination of the tissue biopsy is essential.

The purpose of this study is to know the accuracy of B Scan and CT scan in diagnosing orbital mass pathology which may influence the surgical strategy and later confirming the diagnosis with biopsy.

\section{Materials \& Methods}

Setting: This study is done in a tertiary eye care hospital, Duration: over a period of 18 months.

Type of study: Cross sectional study

Inclusion criteria: Patients with space occupying lesion of the orbit, in the age group of 1 to 70 years were included in the study.

Exclusion criteria: Terminally ill patients and the patients not willing to participate in the study were excluded.

\section{Original Research Article}

Sample size: The study population consisted of 30 patients, suspected to have orbital involvement.

The study was started after obtaining permission from the ethical committee.

Data collection procedure: Routine proptosis workup is done for those patients by appropriate laboratory and radiological investigations and presumptive clinical diagnosis is made. Informed consent of all the patients was taken.

Detailed history of all patients including complaints, family history, ocular history, drug history and systemic history.

Proptosis assessment was done. Patients were then examined in detail, best corrected visual acuity (BCVA) with snellen's chart, slit lamp examination, fundus examination was done.

All the patients were subjected to B scan, CT scan and biopsy. Findings in these tests are correlated with each other.

\section{Results}

In the present study, maximum number of cases who came to department was between 0-10 age group, followed by 11-20 age groups. Least number of cases was in the age group of 61-70.

Age wise distribution of cases with orbital masses is as follows, 0-10 years (23.33\%), 1120 years (20\%) 21-30 years (10\%), 3140 years $(10 \%), 31-40$ years $(10 \%), 41-50$ years $(16.67 \%), 51-60$ years $(13.33 \%)$, and $61-70$ years $(6.67 \%) .20$ cases $(66.67 \%)$ were males and 10 cases $(33.33 \%)$ were females. Most common symptom with which patients presented was protrusion of eyeball (66.67\%) followed by visible mass (30\%), and pain (23.34\%) (Figure 1).

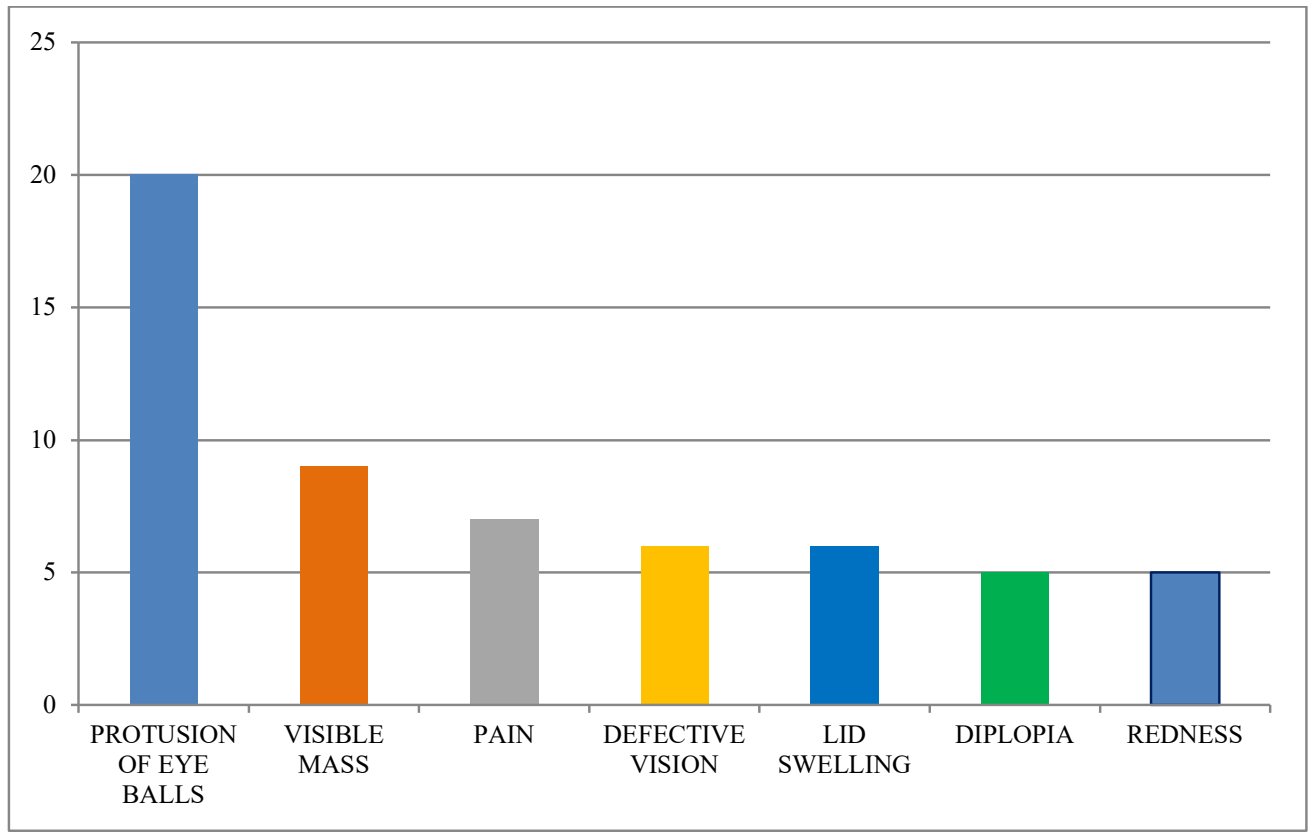

Fig-1: Common symptoms. 
Original Research Article

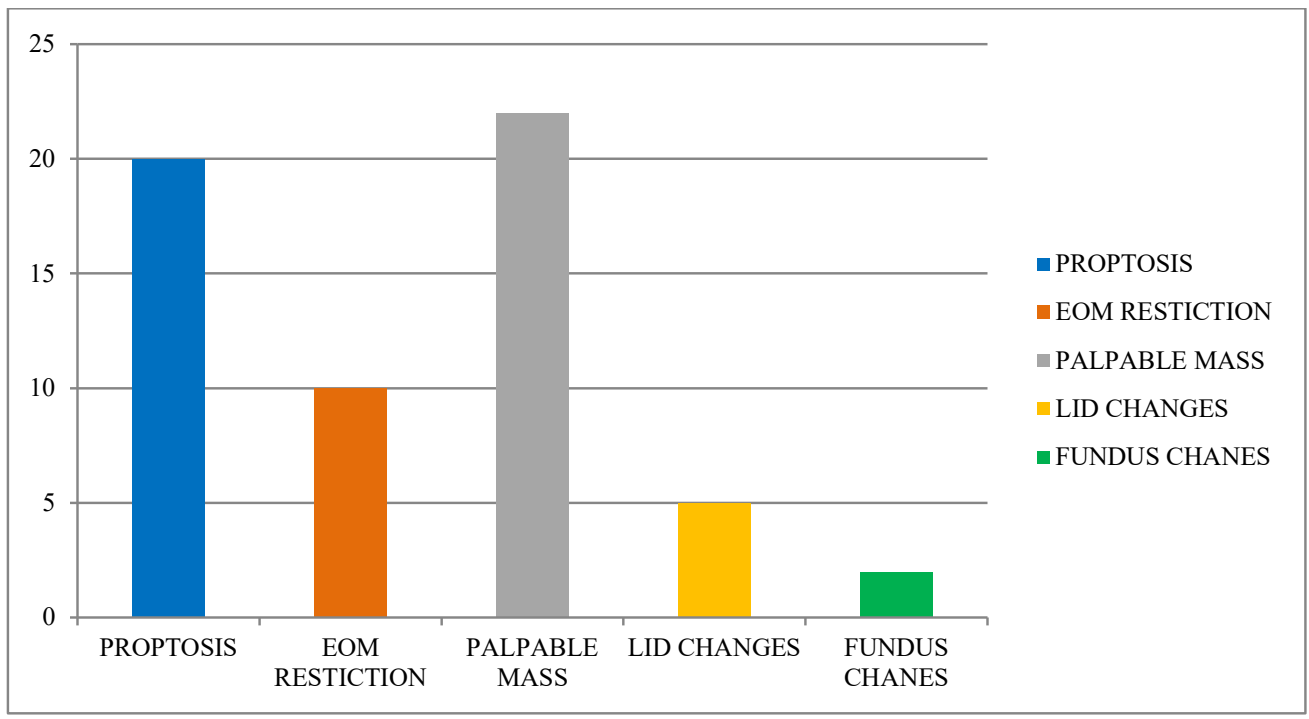

Fig-2: Most common signs.

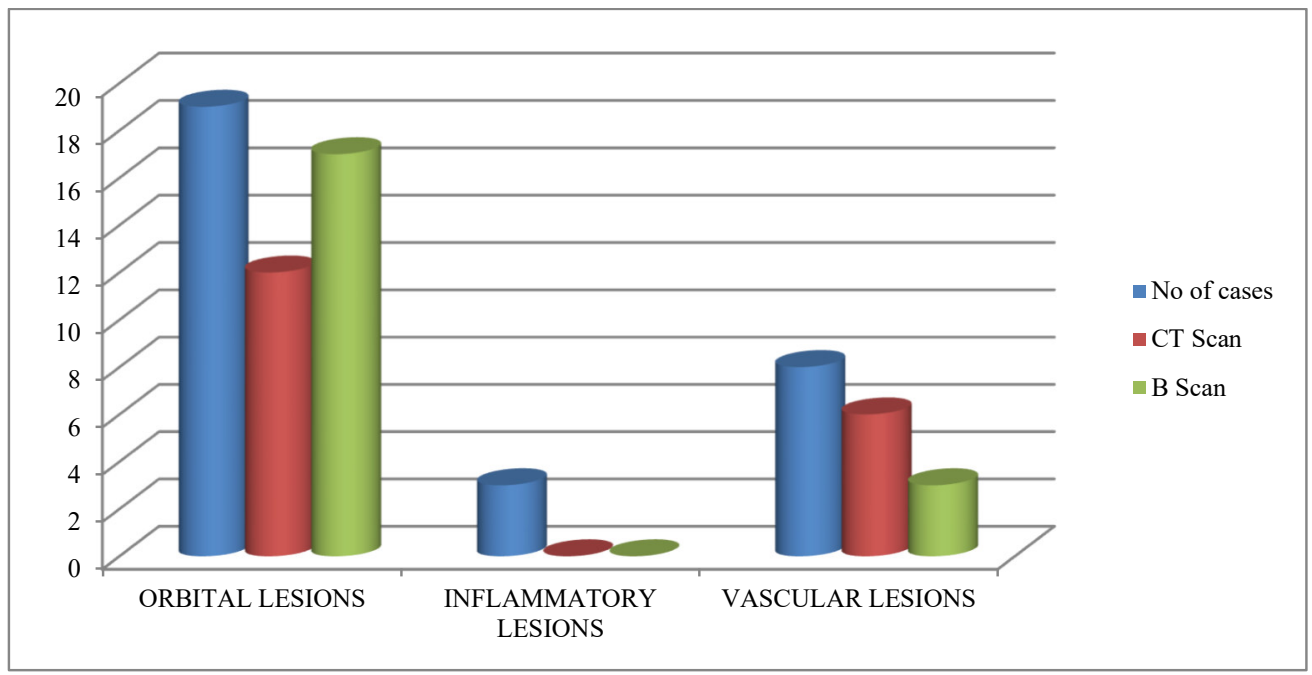

Fig-3: Orbital, inflammatory and vascular lesions.

Most common sign was palpable mass seen in 22 cases $(73.33 \%)$ followed by proptosis in 20 cases (16.67\%) and EOM restriction in 10 cases $(6.67 \%)$ (Figure 2$)$.

22 cases $(73.33 \%)$ were extraconal, followed by 5 cases $(16.67 \%)$ and diffuse in 3 cases $(10 \%)$.

Majority of the cases 8 cases (26.66\%) were lacrimal gland tumors, followed by dermoid cyst ( 5 cases, 16.66\%). All the cases in the present study are divided in to three groups - orbital, inflammatory and vascular lesions. In 30 cases examined, 19 cases $(63.33 \%)$ were orbital lesions followed by vascular lesions 8 cases $(26.67 \%)$ and inflammatory lesions were 3 cases (10\%). In the present study, B-Scan identified $89.47 \%$ of orbital lesions and CT scan identified $63.15 \%$ of orbital lesions.

Among the vascular lesions B-Scan identified all the cases and CT scan identified $75 \%$ of the cases (Figure 3). On comparing the findings of B-Scan, CT Scan and biopsy (biopsy findings being taken as gold standard), B-Scan accurately diagnosed $83.33 \%$ of the cases, where as CT scan diagnosed only $60 \%$ of the cases accurately. Rest of the cases, there was no correlation between the B-Scan/CT scan and biopsy. Out of 30 cases, the diagnosis of B-Scan and CT scan correlated in $66.67 \%$.

Statistical parameters for B-Scan showed sensitivity of $84.62 \%$, specificity of $25 \%$, positive predictive value (PPV) of $83.33 \%$, negative predictive value (NPV) of $8.33 \%$ and accuracy of $53.33 \%$. 
Original Research Article

\section{Discussion}

Orbital pathologies are quite common and pose a diagnostic challenge for clinicians. A wide variety of pathologies are known to affect orbit, including congenital, infective, inflammatory, vascular, neoplastic and degenerative conditions [5]. They require detailed imaging evaluation for diagnosis and to assess the location, extension and type of lesion. Ultrasound is an easily available initial imaging modality which can be performed for the evaluation of these cases. The superficial location and cystic structure of globe render themselves for characterization of these lesions [6].

Orbital tumors may be broadly divided into intraocular and extraocular, which may be further compartmentalized [7].

In the present study, 30 cases were examined for diagnostic accuracy of imaging in extra ocular masses using B scan and CT scan. Among lacrimal gland tumors, B scan was able to identify all8 cases. On B scan, they appeared as well circumscribed hypoechoeic solid lesions involving lacrimal gland area. CT scan was able to identify 5 cases. Benign lesions showed well defined, round to oval lesions that are smooth in outline. Malignant lesion showed irregular margins. On histopathology, out of 8 cases, 5 cases were diagnosed as pleomorphic adenoma and 3 cases were adenoid cystic carcinoma. Out of 5 cases of dermoid cyst, B-Scan identified all 5 cases, whereas CT scan identified 4 cases. B-Scan showed moderately regular internal structure with variable reflectivity and sound attenuation. On CT scan they appeared as well defined, non enhancing mass with smooth margins, cystic and/or solid components. Histopathology showed stratified squamous epithelium lines, having epidermal appendages such as hair follicles, sweat glands, and sebaceous glands. Among the cases of cavernous hemangioma, both B-Scan and CT scan were able to identify all the cases. B-Scan showed smoothly circumscribed lesion with regular moderate to high internal echogenicity. CT scan showed well circumscribed, rounded or oval soft tissue density mass hypoattenuating compared to muscle. On histopathology, they appeared as well- circumscribed masses bounded by a fibrous pseudocapsule. They were composed of dilated large vascular spaces lined by flattened and attenuated endothelial cells.

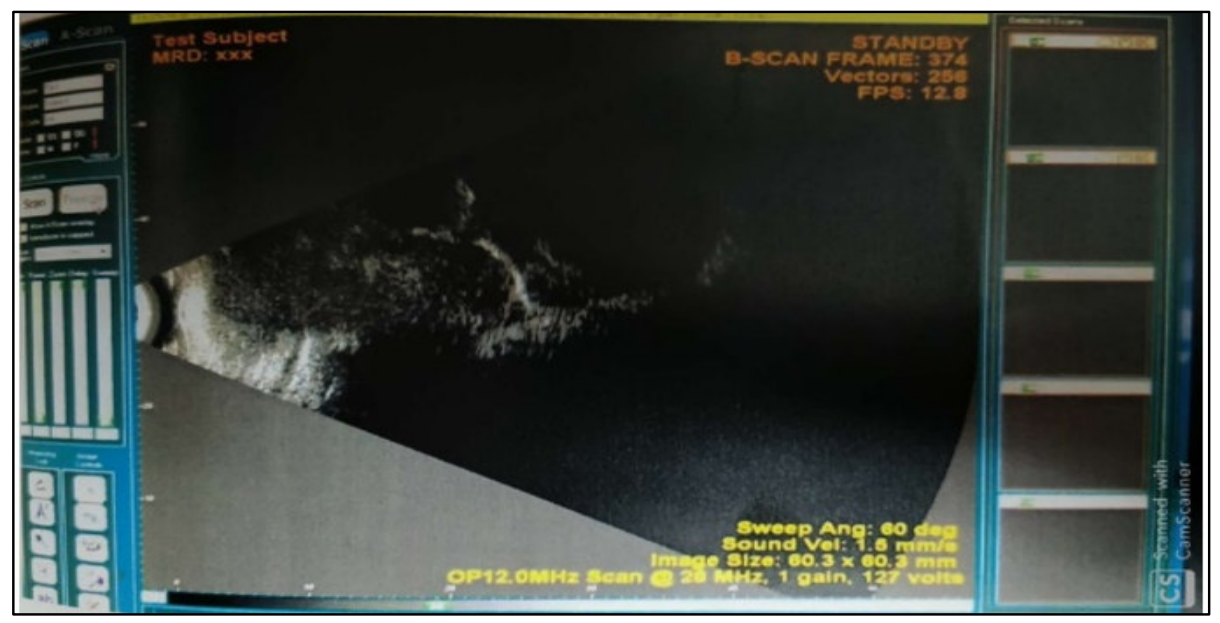

Fig-4: Showing B scan image of lymphoma.

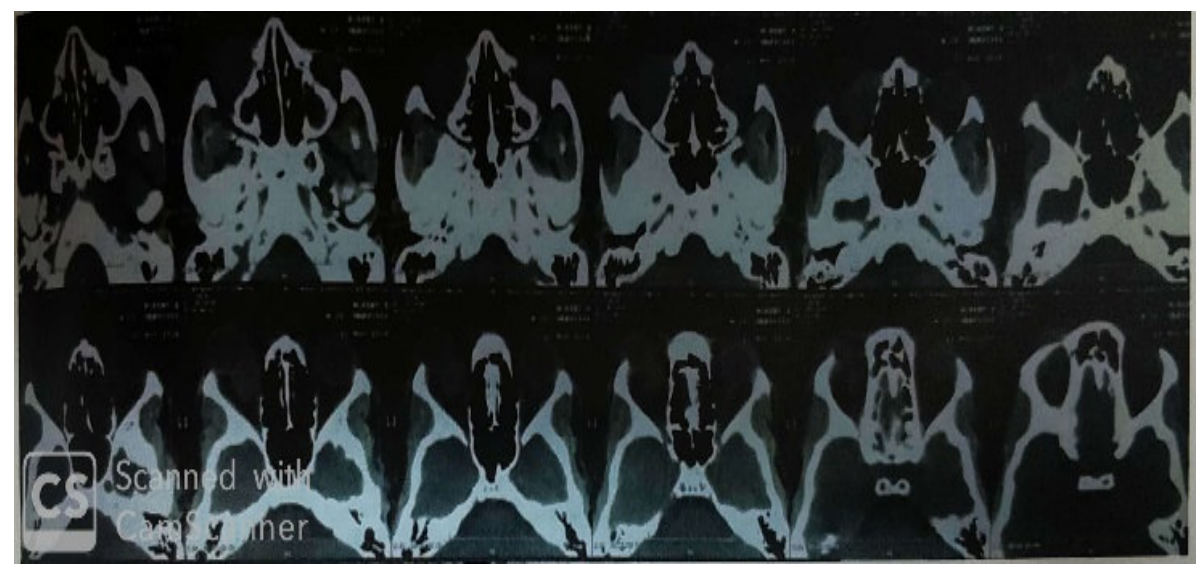

Fig-5: Figure showing CT scan image of lymphangioma. 
Original Research Article

2 cases of lymphangioma were identified during the study. In both cases B-Scan showed irregular outline, borders are moderately well defined. Tumor is not encapsulated and extends diffusely through the orbit. Internal structure is irregular due to mix of high and low reflectivity. CT scan showed lesion with irregular margin, multiloculated, heterogenous lesion and enhance prominently with contrast. On histopathology, they appeared as large lymphatic channels in loose connective tissue stroma, focally disorganized smooth muscle in wall of larger channels, peripheral lymphoid aggregates are seen (Figure 4,5). Optic nerve gliomais demonstrated as smooth fusiform or ovoid mass replacing the normal optic nerve void with low- medium reflectivity, whereas on CT, it is seen as nopdular or fusiform enlargement of the optic nerve with contrast enhancement. On histopathology - low grade pilocyticastrocytomas with round to spindled nuclei and dendrite like cytoplasmic processes, with marked pleomorphism, necrosis, vascular proliferation and tumor cells in pools of mucin are seen. One case of lymphoma identified in the present study was accurately diagnosed by B-Scan, which was well circumscribed with smooth contour, diffuse/irregularly shaped, attached to extraocular muscle, but CT scan demonstrated it as a Pseudotumor. Both B-Scan and CT were unable to identify the case of mucormycosis in the present study. B-Scan identified it as infiltrative mass and CT identified it as carcinoma maxillary antrum involving the orbit.

Ukponmwan CU et al, found $92.3 \%$ correlation between clinical and ultrasonographic diagnosis [8]. SB Adebayo et al, reviewed 29 patients to assess if ocular B-Scan ultrasonography and found $95 \%$ of the patients correlated with the diagnosis [9]. Hafiz MA et al studied 50 cases with B-Scan and found it to be significantly accurate in diagnosing orbital masses including neoplastic and inflammatory conditions [10]. Zhang w et al, examined 288 cases with orbital diseases by using the technique of CDI ultrasound and concluded that CDI can provide the information of colour blood flow in orbital diseases, especially the tumors with rich vascular tissue and orbital vascular diseases, in combination with B-mode ultrasound, CT or MRI [11]. Itani KM et al, found ultrasound to be $100 \%$ successful in diagnosing orbital mass. Accurate diagnosis was made in $78 \%$ of cases with ultrasound, 52\% with clinical and 52\% with radiological examination [12]. Rashmi M nagaraju et al, studied 85 patients of orbital masses, and found B scan sensitivity, specificity, accuracy of $94.2 \%, 99 \%$ and $95.05 \%$ respectively [13]. K Sambasivarao et al, 80 patients presenting with proptosis were evaluated for CT scan orbits. CT diagnosed cases with accuracy of $85 \%$ [14]. JA Akinmoladun et al study included 66 patients of orbital lesions. CT diagnoses of benign and malignant tumors were accurate when compared with histopathological diagnosis in $80.6 \%$ and $96.7 \%$ of the cases respectively [15]. In the present study, B scan and CT scan was able to diagnose $83.33 \%$ and $60 \%$ respectively, where as in Sanjeevsuman et al B-Scan and CT scan was able to diagnose $98.80 \%$ and $53.57 \%$ respectively, in Berger et al B-Scan and CT scan was able to diagnose $95.75 \%$ and $83.33 \%$ respectively.

Limitations of the study: although B-Scan is a better tool in diagnosing the orbital lesions, the findings are variable and dependent on the ophthalmologist. CT-scan is helpful in diagnosing variety of bony and orbital lesions. However constant exposure to the radiation may have adverse effects.

\section{Conclusion}

In the present study, B-Scan appears to be the better diagnosing tool in identifying most of the orbital lesions when compared to the $\mathrm{CT}$ scan. Considering radiation exposure, repeated examination, cost effectiveness and time consumption, B-Scan is advantageous over CT scan in the initial work up and follow up of cases. Although B-Scan is advantageous in initial diagnosis, imaging by $\mathrm{CT}$ scan appears more complete, as it helps in delineating tumor boundaries which helps in planning the surgery. All the authors, (first author, second author, corresponding author and the third author) contributed to the data collection, analysis and in writing and reviewing the article.

\section{What the study adds to the existing knowledge?}

Although histopathological diagnosis is the ultimate in diagnosing the orbital lesions, it was found in the present study that B-scan, if done consistently by the same person, the results are similar to the histopathological diagnoses.

\section{Author's contributions}

Dr. Modini Pandarpurkar: Manuscript preparation

Dr G.V. Shilpa: Statistical analysis

Dr. K.S. Amruthavalli: Concept, statistical analysis

Dr N. Rajesh Kumar: Manuscript preparation

Funding: No funding sources

Conflict of interest: None declared

Ethical Approval: This study was approved by the Institutional Ethics Committee

\section{References}

1. Mallajosyula S, Surgical atlas of orbital diseases. $1^{\text {st }}$ ed. Jaypee Brothers Med Publ LTD. 2006;104-108.

2. Khan SN, Sepahdari AR. Orbital masses: CT and MRI of common vascular lesions, benign tumors, and malignancies. Saudi J Ophthalmol. 2012; 26 (4): 373-83. doi: 10. 1016/j.sjopt.2012.08.001. 


\section{Original Research Article}

3. Southern S. Ultrasound of the eye. Australas J Ultrasound Med. 2009; 12(1): 32-37. doi: 10.1002/j.2205-0140.2009. tb00005.x. Epub 2015 Dec 31.

4. Naik MN, Tourani KL, Sekhar GC, Honavar SG. Interpretation of computed tomography imaging of the eye and orbit. A systematic approach. Indian J Ophthalmol. 2002; 50(4):339-353.

5. Bran A, Tripati R, Tripati B. Wolfs anatomy of the eye and the orbit, $8^{\text {th }}$ edition. Chapman $\&$ Hall India,1997, page 11-23.

6. De La Hoz Polo M, Torramilans Lluís A, Pozuelo Segura O, Anguera Bosque A, Esmerado Appiani C, Caminal Mitjana JM. Ocular ultrasonography focused on the posterior eye segment: what radiologists should know. Insights Imaging. 2016;7(3):351-64. doi: 10.1007/s13244016-0471-z. Epub 2016 Feb 24.

7. Choudary N, Verma SR, Gupta PK, Sharma S, Awana A. Evaluation of orbital and ocular lesions on sonography. IOSR J Dental Med Sci. 2017;16(6):50-58. doi: 10.9790 / 0853-1606015058.

8. Ukponmwan CU, Marchien TT. Ultrasonic diagnosis of orbito-ocular diseases in Benin City, Nigeria. The Nigerian postgraduate Med J. 2001;8(3):123-126.

9. Hafiz MA, Mustansar MW. Ultrasound of the eye and orbit. Can J Med. 2011;2(1):39.
10. Glasier CM, Brodsky MC, Leithiser RE Jr, Williamson SL, Seibert JJ. High resolution ultrasound with Doppler: a diagnostic adjunct in orbital and ocular lesions in children. Pediatr Radiol. 1992;22(3):174-178. doi: 10.1007/ bf 02012488 .

11. Scott IU, Smiddy WE, Feuer WJ, Ehlies FJ. The impact of echography on evaluation and management of posterior segment disorders. Am J Ophthalmol. 2004;137(1):24-29. doi: 10.1016/s0002-9394(03)00910-3.

12. Itani KM, Frueh B, Nelson C. The value of orbital echography in orbital practice. Ophthalmic Plast Reconstr Surg. 1998; 14(6): 432-435. doi: 10.1097/00002341199811000-00007.

13. Nagaraju RM, Gurushankar G, Bhimarao3, Kadakola B. Efficacy of High Frequency Ultrasound in Localization and Characterization of Orbital Lesions. J Clin Diagn Res. 2015; 9(9): TC01-TC06. doi: 10.7860/JCDR/2015/ 13021. 6428. Epub 2015 Sep 1.

14. Sambasivarao K, Ushalatha B. Diagnostic role of CT in the evaluation of proptosis. IOSR J Dent Med Sci. 2015; 14(4): 25-31. doi: 10.9790/0853-14492531.

15. Akinmoladun JA, Adeyinka AO, UchenduandO, Akinmoladun. Evaluation of the effectiveness of computed tomography in the diagnosis of orbital tumors in Ibadan, Nigeria, J West AfrColl Surg. 2013;3(3):46-62.

\section{How to cite this article?}

Pandarpurkar M, Shilpa G.V, Amruthavalli K.S, N. Rajesh Kumar. Correlation of B-scan, CT scan and biopsy findings in orbital masses (space occupying lesions). Trop J Ophthalmol Otolaryngol.2019;4(8):457-462.doi:10.17511/jooo.2019.i08.02 